\title{
PID Controller Design of Nonlinear System using a New Modified Particle Swarm Optimization with Time-Varying Constriction Coefficient
}

\author{
Alrijadjis ${ }^{1}$, Shenglin Mu$^{2}$, Shota Nakashima ${ }^{3}$, Kanya Tanaka ${ }^{4}$ \\ 1)PoliteknikElektronikaNegeri Surabaya, 2)Hiroshima National College of Maritime \\ Technology, 3),4)Yamaguchi University \\ 1)Jl. Raya ITS, Keputih, Sukolilo, Surabaya, Indonesia, Telp. +62(31)5947280, 2)4272- \\ 1 Higashino, Osakikamijima, Toyota District, Hiroshima 725-0231, Japan, Telp. +81- \\ 846-65-3101, 3),4)2-16-1 Tokiwadai, Ube-shi 755-8611, Japan, Telp. +81-836-85- \\ 9005 \\ E-mail: 1)alrijadjis@pens.ac.id, 2)mshenglin@hiroshima-cmt.ac.jp, \\ 3)S-naka@yamaguchi-u.ac.jp, ${ }^{4) k t a n a k a @ y a m a g u c h i-u . a c . j p ~}$
}

\begin{abstract}
The proportional integral derivative (PID) controllers have been widely used in most process control systems for a long time. However, it is a very important problem how to choose PID parameters, because these parameters give a great influence on the control performance. Especially, it is difficult to tune these parameters for nonlinear systems. In this paper, a new modified particle swarm optimization (PSO) is presented to search for optimal PID parameters for such system. The proposed algorithm is to modify constriction coefficient which is nonlinearly decreased time-varying for improving the final accuracy and the convergence speed of PSO. To validate the control performance of the proposed method, a typical nonlinear system control, a continuous stirred tank reactor (CSTR) process, is illustrated. The results testify that a new modified PSO algorithm can perform well in the nonlinear PID control system design in term of lesser overshoot, rise-time, settling-time, IAE and ISE.
\end{abstract}

Keywords: PID controller, Particle Swarm Optimization (PSO), constriction factor, nonlinear system.

\section{INTRODUCTION}

The proportional - integral - derivative (PID) controller operates the majority of the control system in the world. It has been reported that more than $90 \%$ of the controllers in the industrial process control applications are of PID type as no other controller match the simplicity, clear functionality, applicability and ease of use offered by the PID controller [2]. The PID controller is used for a wide range of problems like motor drives, automotive, 
flight control, instrumentation, etc. However, it is a main problem how to choose PID parameters. PID controllers can provide robust and reliable performance for most systems if the PID parameters are tuned properly. Various tuning methods are explained in [1-3]. But, these conventional tuning less optimal because its use assumption that the system have minimum dynamic characteristic, linear (LTI), no-disturbance. In fact, industrial processes are nonlinear, time-varying and complex [4], [5].

Several optimization methods for tuning PID parameters have been developed, including LQR method, the results of this method is optimal, but requires complex mathematical calculations [6]. To avoid the complex mathematical calculaton, many researchers have developed intelligent softcomputing for optimal tuning PID parameters, such as Genetic Algorithm (GA), Tabu Search (TS) and Simulated Annealing (SA) have recently received great attention for searching global optimal solution and achieving high efficiency [7],[8]. GA method is usually faster than TS and SA methods because of employing parallel search techniques. Though, the GA method has been employed successfully for global optimization, recent research has identified some deficiencies in GA performance. This degradation in efficiency is apparent in applications with highly epistatic objective function (where the parameters being optimized are highly correlated), the crossover and mutation operations cannot ensure better fitness of offspring because population chromosomes have similar structure and their fitness are high toward the end of the process [9]. To overcome GA difficulties, Kennedy and Eberhartin their paper proposed PSO method. PSO is one of the modern heuristic algorithm developed through simulation of a simplified social system and has been introduced in various application fields in recent years. Generally, it is characterized as a simple concept, easy to implement and computationally efficient.

Application of PSO method for tuning PID parameters with a variety of plant models, including nonlinear plant, have shown better results than previous methods [2], [10], [11]. Although PSO has the characteristics of fast convergence, good robustness, strong commonality, and has been successfully applied in many areas, it has the shortcomings of premature convergence, low searching accuracy and iterative inefficiency, especially the problems involving multiple peak values, and it is likely to fall in local optima. In order to overcome the aforementioned limitations, many researchers have attempted to improve the PSO algorithm. Some of these include the random inertia weight PSO (PSO-RIW), linear decreased weight PSO (PSO-LDW), nonlinear decreased weight PSO (PSO-NDW), variable constriction coefficient PSO (PSO-VCF), Genetic Algorithm PSO (GA-PSO), different evolutional PSO (DE-PSO), dynamic multi-point detecting PSO, binary PSO, self adaptive PSO (SA-PSO), knowledge based PSO (KB-PSO), and so on [12]. These improved PSO algorithms have enriched the PSO theory and they are convenient to apply to various areas. Many improvement strategies based on adjustment of inertia weight because this parameter is very influential [13]. 
In this work, a new modified PSO with time-varying nonlinear decreased constriction coefficient was applied to optimize the PID parameters for nonlinear system. To show the effectiveness of our proposed method, the step responses of closed loop system were compared with that of the existing methods (ZN, PSO-CFA and PSO-VCF). The reminder of this paper is organized as follows. Section 2 introduces the particle swarm optimization algorithm. Section 3 describes the proposed PSO with timevarying nonlinear decreased constriction coefficient. Application of our proposed algorithm to optimal PID tuning is described in section 4. Section 5 describes result and discussion. Finally, section 6 concludes the paper.

\section{PARTICLE SWARM OPTIMIZATION ALGORITHM}

PSO is one of the optimization techniques and a kind of evolutionary computation technique. The method has been found to be robust in solving problems featuring nonlinearity and no- differentiability, multiple optima, and high dimensionality through adaptation, which is derived from the social-psychological theory [9]. The technique is derived from research on swarm such as fish schooling and bird flocking. According to the research results for a flock of birds, birds find food by flocking (not by each individual). The observation leads the assumption that every information is shared inside flocking. Moreover, according to observation of behavior of human groups, behavior of each individual (agent) is also based on behavior patterns authorized by the groupssuch as customs and other behavior patterns according to the experiences by each individual. The assumption is a basic concept of PSO. In the PSO algorithm, instead of using evolutionary operators such as mutation and crossover, to manipulate algorithms, for a dvariabled optimization problem, a flock of particles are put into the $d$ dimensional search space with randomly chosen velocities and positions knowing their best values so far (Pbest) and the position in the $d$ dimensional space. The velocity of each particle, adjusted according to its own flying experience and the other particle's flying experience. For example, the $i$-th particle is represented as $x_{i}=\left(x_{i, 1}, x_{i, 2}, \ldots x_{i, d}\right)$ in the d-dimensional space. The best previous position of the $i$ th particle is recorded and represented as pbest $_{i}=\left(\right.$ pbest $_{i, 1}$, pbest $_{i, 2}, \ldots$, pbest $\left._{i, d}\right)$.

The index of best particle among all of the particles in the group is gbest $_{\mathrm{d}}$. The velocity for particle $i$ is represented as $v_{i}=\left(v_{i, 1}, v_{i, 2}, \ldots v_{i, d}\right)$. The modified velocity and position of each particle can be calculated using the current velocity and the distance from pbest $t_{i, d}$ to gbest $_{d}$ as shown in the following formulas:

$$
\begin{aligned}
& v_{i, m}^{(t+1)}=w \cdot v_{i, m}^{(t)}+c_{1} * R *\left(\text { pbest }_{i, m}-x_{i, m}^{(t)}\right)+c_{2} * R *\left(\text { gbest }_{m}-x_{i, m}^{(t)}\right) \\
& x_{i, m}^{(t+1)}=x_{i, m}^{(t)}+v_{i, m}^{(t+1)}
\end{aligned}
$$




$$
\begin{aligned}
& i=1,2, \ldots, n \\
& m=1,2, \ldots, d
\end{aligned}
$$

where :

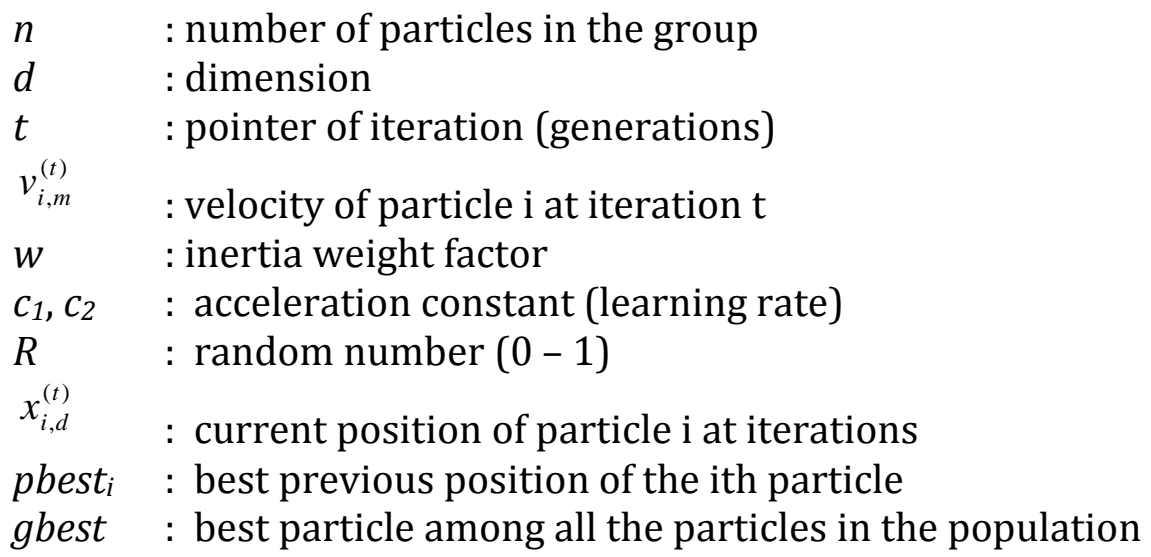

\section{PROPOSED MODIFIED PSO}

The inertia weight $w$ plays an important role in the convergence of the PSO algorithm to the global optimal solution and hence has an influence on the time taken for a simulation run. Recall here that the weight factor is used to control the influence of the previous history of the particle velocities on both the current velocity and the local and global exploration capabilities of the PSO algorithm. It thus follows that the reason for using a linearly decreasing-in-time inertia weight parameter $w$ is that larger values of $w$ tend to be used at the start of the search to enable the PSO algorithm to explore globally the solution space, whereas smaller values of $w$ are used toward the end of the search to enable the PSO algorithm to explore locally around the global optimum before finally homing in onto it.

Recent works in [14] indicate that the use of a "constriction factor" may be necessary to insure convergence of the PSO. A simplified method of incorporating a constriction factor is represented in:

$$
v_{i, m}^{(t+1)}=K \cdot\left[v_{i, m}^{(t)}+c_{1} * R *\left(\text { pbest }_{i, m}-x_{i, m}^{(t)}\right)+c_{2} * R *\left(\text { gbest }_{m}-x_{i, m}^{(t)}\right)\right]
$$

where $K$ is a function of $c_{1}$ and $c_{2}$ as illustrated by the following equation [9]:

$$
K=\frac{k}{\left|2-\varphi-\sqrt{\varphi^{2}-4 \varphi}\right|}
$$

where $k=2, \phi=c_{1}+c_{2}$, and $\phi>4$. In [14], the performance of PSO using an inertia weight was compared with the PSO performance using a constriction factor. It was concluded that the best approach is to use a constriction factor while limiting the maximum velocity $v_{\max }$ to the dynamic 
range of the variable $x_{\max }$ in each dimension. It was also shown in [14] that this approach provides a performance superior to any similar technique reported in the literature.

Ali T. Al Awami proposed a PSO with variable constriction factor for adaptive channel equalization by introducing a time-varying linearlydecreasing $K$, instead of a fixed one. This is done by adjusting $k$ at every iteration according to the following formula [15]. He calls his method as PSO VCF (PSO Variable Constriction Factor).

$$
k_{n}=k_{\min }+\left(k_{\max }-k_{\min }\right) \frac{m-n}{m-1}
$$

Where $m$ is the maximum number of iterations and $n$ is the current iteration.

Building on the result in [15], we propose PSO NDCC (PSO Nonlinear Decreased Constriction Coefficient) method in this work, a new modification of the constriction factor-based technique for optimal tuning of PID parameters by introducing a time-varying nonlinearly-decreasing $K$. This is done by adjusting $k$ at every iteration according to the following recursion:

$$
k_{n}=k_{\min }+\left(k_{\max }-k_{\min }\right)\left(\frac{m-n}{m-1}\right)^{x}
$$

Where $x$ is the index modulation.

\section{PSO-NDCC BASED PID CONTROLLER}

Design of PSO NDCC based-PID controller for plant is shown in Fig. 1. In this system, three PID parameters, i.e., the proportional gain Kp, integral gain $\mathrm{Ki}$, and derivative gain $\mathrm{Kd}$, will be tuned optimally by IPSO algorithm.

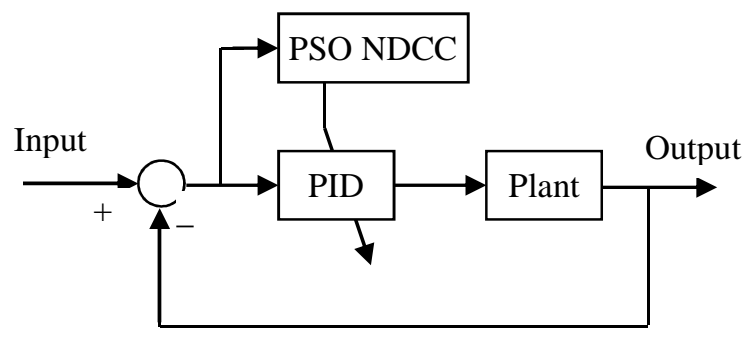

Figure 1. The structure of MPSO-PID

Performance index that is used to estimate the PID parameters are given as follows:

$$
I A E=\int_{0}^{T}|e(t)| d t
$$




$$
I S E=\int_{0}^{T} e^{2}(t) d t
$$

where IAE is an integral absolute error and ISE is an integral square error.

The main concept of PID controller tuning for on-line system is tuning the PID parameter of each sampling time. The objective function or fitness function that will be optimized is expressed as follows:

$$
J(i)=\alpha \cdot(\operatorname{IAE}(i)+\operatorname{ISE} E(i))+\beta \cdot \mid O(i)
$$

where :

$$
\begin{array}{ll}
\alpha, \beta & : \text { improvement factor } \\
0 & : \text { overshoot }
\end{array}
$$

Flowchart of the IPSO-PID controller is shown in Fig. 2.

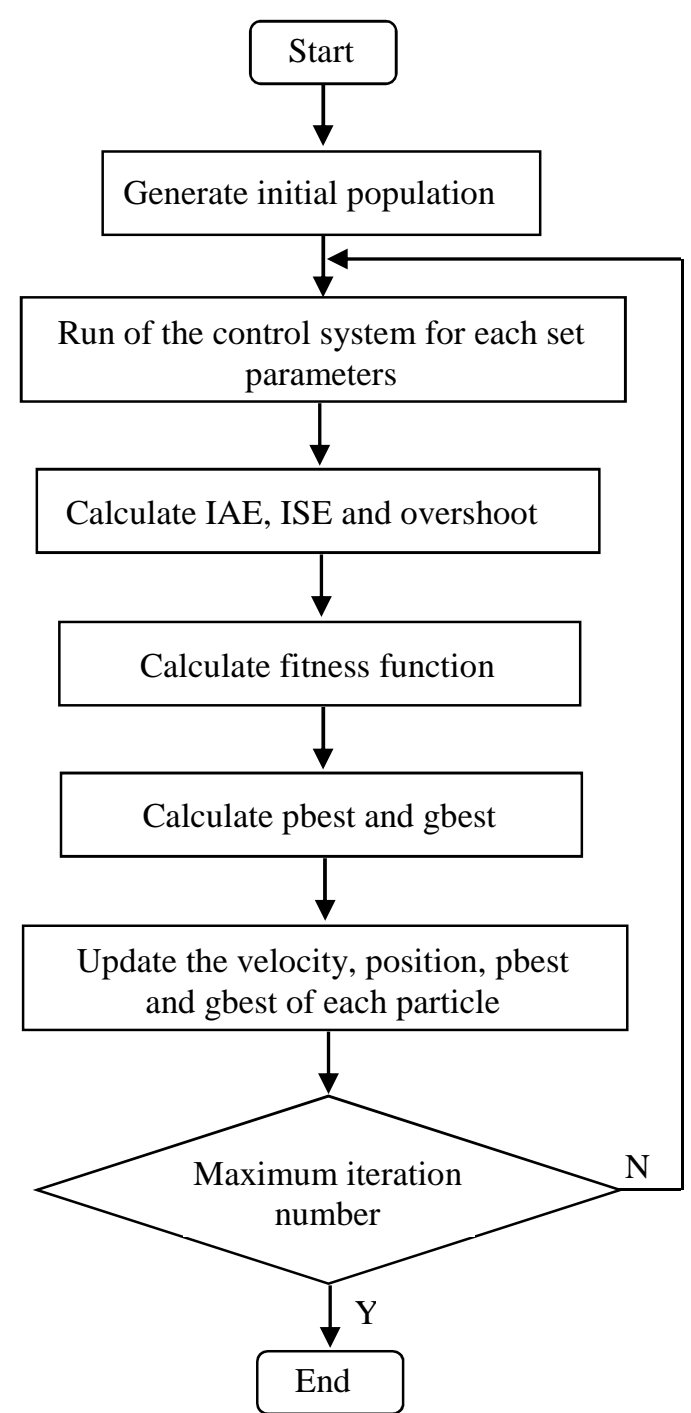

Figure 2. Flowchart of PSO algorithm 


\section{RESULTS AND DISCUSSION}

In order to illustrate the between the PID tuning process with PSONDCC and the other methods (ZN, PSO-CFA, and PSO-VCA), the following model (10) is taken from [16]. This model is a CSTR process model and it is typical of nonlinear systems.

$$
G(s)=\frac{(1-5 s)}{(1+10 s)(1+20 s)}
$$

Using three different methods (PSO-CFA, PSO-VCF and PSO-NDCC), tuning process is applied to this model. Because of the probabilistic nature of the PSO algorithm, tuning process was run five times for each method.

The strategy approach to a value of constriction coefficient for each method is shown in Fig. 3. In this experiment, we used $\mathrm{k}_{\max }=2, \mathrm{k}_{\min }=1$ and $\mathrm{x}$ $=5$ for PSO VCF and PSO NDCC.

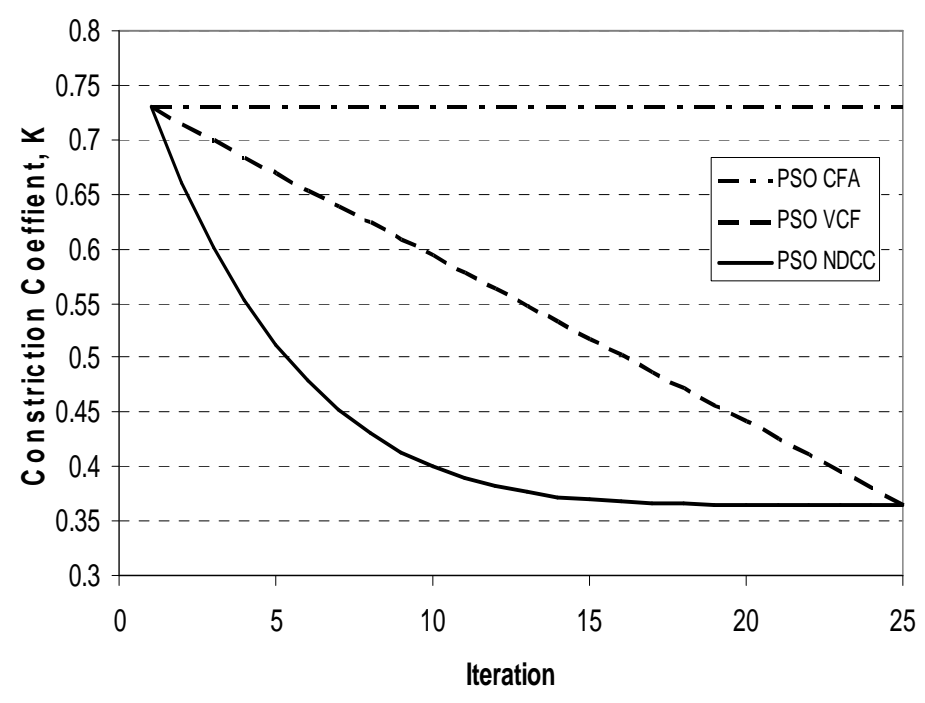

Figure 3. Strategy approach of $\mathrm{K}$

To examine effectiveness and ability to find global optima, we used three categories of population size, the first, small population size $(n=5)$, the second, medium size population $(n=25)$ and the last large population size $(n$ =50). Other used parameters are:

1. Iteration number, iter $=25$

2. Cognitive constant, $\mathrm{c}_{1}=2.05$

3. Social constant, $\mathrm{c}_{2}=2.05$

To evaluate the general performance of system, the following equation (11) is taken from [17].

$$
I P_{\text {total }}=M p+t r+t s+I A E+I S E
$$


Where $\mathrm{IP}_{\text {total }}$ is index performance total, $\mathrm{Mp}$ is maximum overshoot in percent, tr is rise-time in second, ts is settling-time in second, IAE is integral absolute error, and ISE is integral square error. The smallest of IP shows that the performance is best. A comparison of the average of index performance from five running is tabulated as given in Table 1-3. It is found very clearly that our proposed method has a smallest IP in all of particle number and its mean that PSO NDCC can improve performance of system. IPc is the ratio of IP value to maximum IP of ZN method.

Table 1. Comparison of IP (particle number, $n=5$ )

\begin{tabular}{|c|c|c|c|c|}
\hline \multirow{2}{*}{ ITEM } & \multirow{2}{*}{$\mathrm{ZN}$} & \multicolumn{3}{|c|}{$\mathrm{n}=5$} \\
\cline { 3 - 5 } & & PSO-CFA & PSO-VCF & PSO-NDCC \\
\hline IPave & 168.532 & 157.1003 & 98.4065 & 92.4084 \\
\hline IPc & 1 & 0.9322 & 0.5839 & 0.5483 \\
\hline
\end{tabular}

Table 2. Comparison of IP (particle number, $n=25$ )

\begin{tabular}{|c|c|c|c|c|}
\hline \multirow{2}{*}{ ITEM } & \multirow{2}{*}{$\mathrm{ZN}$} & \multicolumn{3}{|c|}{$\mathrm{n}=25$} \\
\cline { 3 - 5 } & & PSO-CFA & PSO-VCF & PSO-NDCC \\
\hline IPave & 168.532 & 124.7325 & 96.1711 & 76.9115 \\
\hline IPc & 1 & 0.7401 & 0.5706 & 0.4564 \\
\hline
\end{tabular}

Table 3. Comparison of IP (particle number, $n=50$ )

\begin{tabular}{|c|c|c|c|c|}
\hline \multirow{2}{*}{ ITEM } & \multirow{2}{*}{$\mathrm{ZN}$} & \multicolumn{3}{|c|}{$\mathrm{n}=50$} \\
\cline { 3 - 5 } & & PSO-CFA & PSO-VCF & PSO-NDCC \\
\hline IPave & 168.532 & 66.033 & 62.1632 & 51.3457 \\
\hline IPc & 1 & 0.3918 & 0.3689 & 0.3047 \\
\hline
\end{tabular}

A comparison of time domain specifications maximum overshoot, risetime, settling-time, IAE and ISE are tabulated as given in Table 4. As can be seen that PSO NDCC significantly reduce the overshoot. Rise-time, settlingtime, IAE and ISE have also improved. Henceforth, outperforms that of the conventionally Ziegler-Nichols method.

Among five runs for each method, the best result for step input is shown in Fig.4. It is found clearly that PSO NDCC outperform the previous methods. All of time domain specifications of PSO NDCC are better than previous methods.

Figure 5 shows the result of convergence characteristic for our proposed method and previous methods. It is found that our proposed method have quick convergence better than PSO CFA and PSO VCF at $9^{\text {th }}$ iteration. Also, PSO NDCC method has a lowest fitness value. It's mean that PSO NDCC can improve the final accuacy and the convergence speed of PSO. 
Table 4. Comparison of time domain specifications

\begin{tabular}{|c|c|c|c|c|}
\hline \multirow{2}{*}{ Item } & \multicolumn{4}{|c|}{ Tuning methods } \\
\cline { 2 - 5 } & ZN & $\begin{array}{c}\text { PSO } \\
\text { CFA }\end{array}$ & $\begin{array}{c}\text { PSO } \\
\text { VCF }\end{array}$ & $\begin{array}{c}\text { PSO } \\
\text { NDCC }\end{array}$ \\
\hline $\mathrm{Kp}$ & 3.5294 & 2.5643 & 3.3482 & 5.6145 \\
\hline $\mathrm{Ki}$ & 0.2101 & 0.0664 & 0.0770 & 0.1013 \\
\hline $\mathrm{Kd}$ & 14.8235 & 5.2050 & 15.9474 & 27.2567 \\
\hline $\mathrm{EsS}$ & 0 & 0 & 0 & 0 \\
\hline $\mathrm{Mp}$ & 52.73 & 19.55 & 6.27 & 3.4 \\
\hline $\mathrm{Tr}$ & 6.522 & 9.615 & 9.925 & 3.641 \\
\hline $\mathrm{Ts}$ & 68.5 & 74.5 & 37.27 & 20.85 \\
\hline IAE & 23.92 & 20.65 & 14.53 & 10.14 \\
\hline ISE & 16.532 & 14.7 & 12.74 & 11.74 \\
\hline IPtotal & 168.532 & 139.015 & 80.735 & 49.771 \\
\hline
\end{tabular}

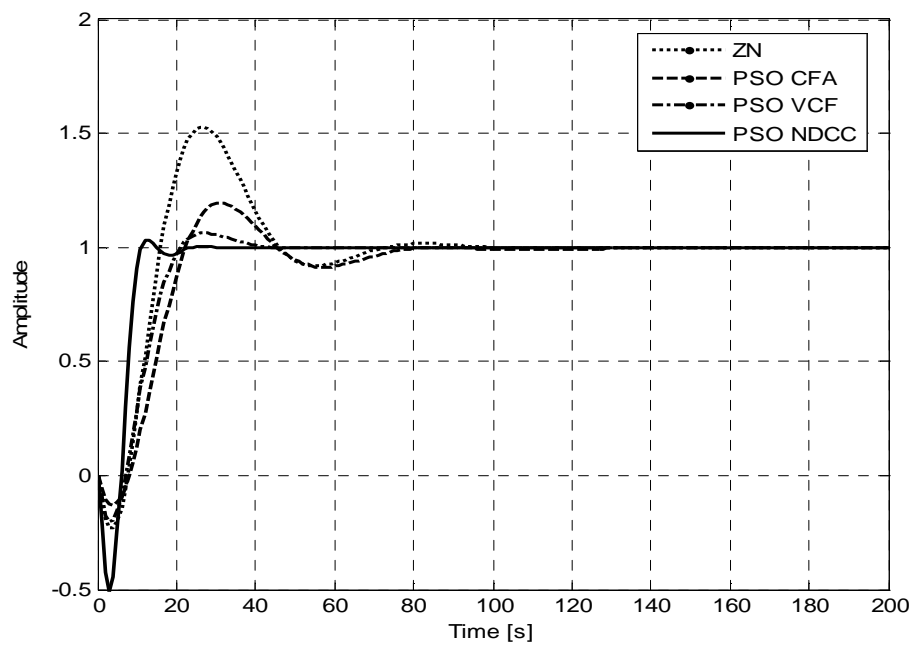

Figure 4. Unit step response

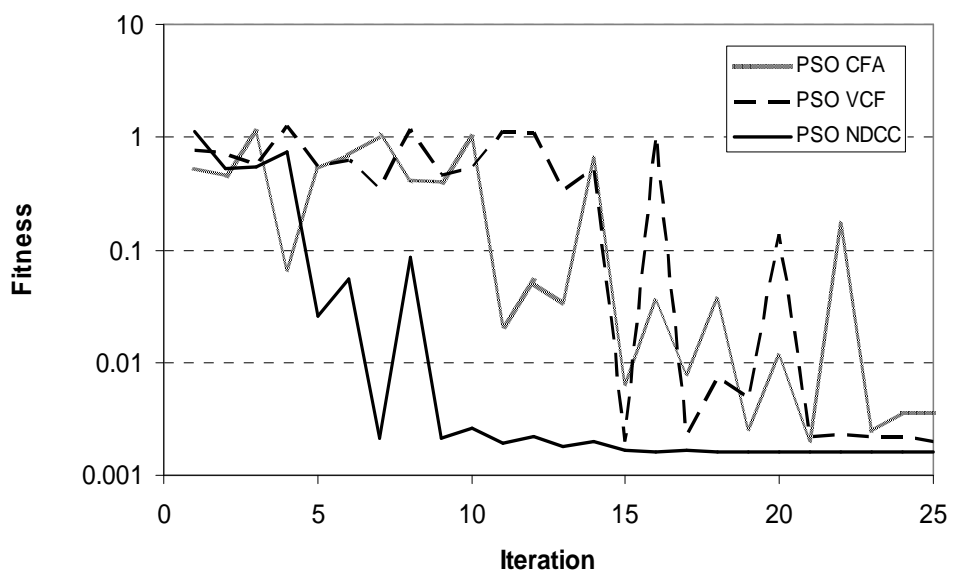

Figure 5. Convergence characteristic 


\section{CONCLUSIONS}

In this paper, a new modified PSO or we call as PSO NDCC for nonlinear system is proposed. It is shown analytically and graphically that there is a substantial improvement in the time domain specification is term of lesser overshoot, rise-time, settling-time, IAE and ISE. Also, our proposed method improved the accuracy searching and the speed convergence.

\section{REFERENCES}

[1] Li Xu-zhou, Yu Fei, Wang You-bo,PSO Algorithm Based Online Selftuning of PID Controller, International Conference on Computational Intelligence and Security, 2007

[2] S.M. Giriraj Kumar, Deepak Jayaraj, Anoop R. Kishan,PSO Based Tuning of a PID Controller for a High Performance Drilling Machine, International Journal of Computer Application (0975-8887), Volume 1, No. 19,2010

[3] P. Cominos, N. Munro,PID Controller: recent tuning methods and design to specification, IEE Proceeding Control Theory and Applications, pp.46-53, 2002

[4] K. Astrom, T. Hagglund, The Future of PID Control, Control Engineering Practice Vol. 9, pp. 1163-1175, 2001

[5] Halevi. Y, Palmor. Z.J, Efrati. T, Automatic Tuning of Decentralized PID Controller for MIMO Processes, Journal of Process Control, Vol. 72, pp. 119-128, 1997

[6] G. Yu, R. Hwang,Optimal PID Speed Control of BLDC motor using LQR Approach, Proceeding IEEE Int. Conf. System, Man and Cybernetics, pp. 473-478, 2004

[7] Zhou. G, J. Birdwell,Fuzzy Logic-based PID Auto-tuner Design Using Simulated Annealing, Proceeding of the IEEE/IFAC Joint Symposium, Computer Aided Control System Design, pp. 67-72, 1994

[8] Haupt. R.L, S.E Haupt, Practical Genetic Algorithm, $2^{\text {nd }}$ ed, John Wiley and Sons Inc, ISBN 0-471-18873-5, 1998

[9] Kennedy J, EberhartC,Particle Swarm Optimization, Proceeding IEEE International Conference on Neural Networks, pp. 1942-1945, 1995

[10] Iraj Hassanzadeh, SalehMobayen,PSO-Based Controller Design for Rotary Inverted Pendulum System, Journal of Applied Sciences 8 (16), pp. 2907-2912, 2008

[11] M. El Said El Telbany,Employing Particle Swarm Optimizer and Genetic Algorithm for Optimal Tuning of PID Controller: A Comparative Study, ICGST-ACSE Journal, Vol. 7, 2007

[12] Di Hu, Ali Sarosh, Yun-FengDong,An Improved Particle Swarm Optimizer for Parametric Optimization of Flexible Satellite Controller, Journal of Applied Mathematics and Computation 217, pp. 8512-8521, 2011 
[13] Ahmad Nickabadi, M. Mehdi Ebadzadeh, Reza Safabakhsh,A Novel Particle Swarm Optimization Algorithm with Adaptive Inertia Weight, Journal of Applied Soft Computing 11, pp. 3658-3670, 2011

[14] R.C Eberhart, Y. Shi,Comparing Inertia Weight and Constriction Factors in Particle Swarm Optimization, Proc. 2000 Congress on Evolutionary Computation, pp. 84-88, 2000

[15] Ali T. Al-Awami, A.Zerguine, L. Cheded, A.Zidouri, WaleedSaif,A New Modified Particle Swarm Optimization Algorithm for Adaptive Equalization, Journal of Digital Signal Processing 21, pp. 195-207, 2011

[16] O. Lequin, M. Gevers, M. Mossberg, E. Bosmans, L. Triest,Iterative Feedback Tuning of PID Parameters: Comparison with Classical Tuning Rule, Control Engineering Practice, Vol. 11, pp. 1023-1033, 2003

[17] E.A Gargari, C. Lucas,Design an Optimal PID Controller using Colonial Competitive Algorith, IEEE Congress on Evolutionary Computation, Singapore, 2007 